\title{
Valores normales del test de marcha de 6 minutos en niños y adolescentes sanos: una revisión sistemática y metaanálisis
}

\author{
Normal values of 6-minute walk test in healthy children and adolescents: \\ A systematic review and meta-analysis
}

\author{
Iván Rodríguez-Núñez ${ }^{\mathrm{a}}$, Felipe Mondaca ${ }^{\mathrm{a}}$, Bárbara Casas ${ }^{\mathrm{a}}$, \\ Catalina Ferreira ${ }^{\mathrm{a}}$, Daniel Zenteno ${ }^{\mathrm{b}}$
}

\author{
aLaboratorio de Biología del Ejercicio, Escuela de Kinesiología, Facultad de Ciencias de la Salud, Universidad San Sebastián, \\ Concepción, Chile \\ bDepartamento de Pediatría, Facultad de Medicina, Universidad de Concepción, Chile
}

Recibido el 10 de junio de 2017, aceptado el 08 de septiembre de 2017

\section{Resumen}

Introducción: El test de marcha de 6 minutos (TM6') es una prueba de ejercicio submáximo ampliamente utilizada en el contexto clínico, la cual, permite evaluar la capacidad física en niños sanos y con enfermedades asociadas. Para una adecuada interpretación del TM6' es necesario comparar el valor obtenido con un valor normal apropiado para la población de interés. El objetivo de este trabajo fue analizar los valores de normalidad del TM6', establecer valores de normalidad agrupados y evaluar su asociación con variables antropométricas y raciales en niños y adolescentes sanos. Métodos: Revisión sistemática de la literatura realizada en 6 bases de datos electrónicas. Se incluyeron estudios de corte transversal que reportaran valores normales del TM6' en niños y adolescentes sanos. Los valores normales de la distancia recorrida (DR) fueron ponderados de acuerdo al tamaño muestral y se evaluó su asociación con el género, la edad y continente de procedencia del estudio. Los coeficientes de correlación entre la distancia recorrida y las variables predictoras fueron metaanalizados mediante el modelo de efectos aleatorios. Resultados: A la revisión ingresaron 10 artículos que consideraron 5.352 individuos (51,9\% hombres). El promedio ponderado de la DR fue de 619,8 m existiendo diferencia entre los grupos de edad, género y continente de procedencia. El metaanálisis de efectos aleatorios reveló una moderada asociación entre la DR y las variables estatura, peso y edad. Conclusión: El TM6' se asocia con el género, continente y variables antropométricas. Las variables predictoras determinan un bajo porcentaje de la DR normal en niños y adolescentes.
Palabras clave:

Test de marcha de 6 minutos;

Prueba de esfuerzo;

Niños;

Adolescentes;

Valores de referencia 


\begin{abstract}
Introduction: The 6-minute walk test (SMWT) is a submaximal exercise test widely used in the clinical setting, which allows the assessment of physical capacity in healthy and chronic children. For a proper interpretation of SMWT it is necessary to compare the value obtained by the patient with a normal value appropriate for the studied population. The aim of this study was to analyze the normal values of SMWT, in order to establish grouped normal values and to evaluate their association with anthropometric and racial variables in healthy children and adolescents. Methods: Systematic review performed in 6 electronic databases. We included studies aimed at determining normal values of SMWT in healthy children and adolescents. Normal values of the SMWT were weighted according to the sample size and their association with the gender, age and continent of origin of the study was evaluated. Additionally, the correlation coefficients between the distance during SMWT and the predictor variables were meta-analyzed using the random effects model. Results: The review included 10 articles that included 5352 individuals $(51.9 \%$ men). The weighted average of the distance during SMWT was $619.8 \mathrm{~m}$, there being a difference between the age, gender and continent of origin groups. The meta-analysis of random effects revealed a moderate association between the distance and height, weight and age variables. Conclusion: SMWT is associated with gender, continent and anthropometric variables. However, the predictive variables determine a low percentage of normal walked distance in children and adolescents.
\end{abstract}

Keywords:

Six minute walk test;

Exercise test;

Children;

Adolescents;

Reference values

\section{Introducción}

Los métodos de evaluación de la capacidad física son herramientas necesarias para la evaluación funcional cardiorrespiratoria en niños sanos y aquellos con enfermedades cardiorrespiratorias crónicas ${ }^{1,2}$.

Una de las pruebas más empleadas en el contexto clínico corresponde al test de marcha de 6 minutos (TM6'). El TM6' es un test de ejercicio submáximo sencillo, y de fácil acceso; en el cual, el individuo debe recorrer la mayor distancia posible en un período de $6 \mathrm{~min}^{3}$. El rendimiento durante el TM6' se asocia estrechamente con las actividades de la vida diaria, así como también, permite determinar el deterioro funcional del daño pulmonar en pacientes con enfermedades respiratorias crónicas ${ }^{4,5}$

En el año 2002 la Sociedad Americana del Tórax (ATS) publicó una declaración que contenía las normas generales para la realización del $\mathrm{TM}^{\prime 3}$; lo que promovió su utilización y estandarización, permitiendo su aplicación en diferentes poblaciones con resultados comparables ${ }^{6}$. Así, el TM6' ha sido empleado en individuos con diversas entidades nosológicas, tanto adultos, como niños, en los cuales ha mostrado altos niveles de validez y confiabilidad ${ }^{1,7-10}$.

Para una adecuada interpretación del TM6' es necesario comparar los valores alcanzados por el individuo con valores de normalidad apropiados para la población de interés. Por este motivo, en los últimos años, se han desarrollado una plétora de estudios dirigidos a determinar los valores de normalidad de la distancia recorrida (DR) durante el TM6' en diversas poblaciones y grupos etarios ${ }^{11-21}$. Estos estudios han sido analizados en revisiones previamente realizadas, en las cuales, se destaca la amplia heterogeneidad entre los artículos y la inconsistente capacidad predictiva de las co-variables analizadas ${ }^{2,6}$. Los estudios publicados concuerdan en que las distintas modificaciones que se han realizado al protocolo de ejecución estandarizado de la prueba son una importante fuente de variabilidad entre los estudios, impidiendo establecer con certeza valores de normalidad globales y la real magnitud de asociación entre el TM6' y otras variables asociadas ${ }^{2,6}$. En consecuencia, la revisión y análisis de los valores de referencia de la DR durante el TM6' realizado exclusivamente de acuerdo a la norma ATS es todavía materia de estudio.

La presente revisión sistemática tiene como objetivo analizar los valores de normalidad durante el TM6 ejecutado bajo el estándar ATS, determinar valores de normalidad ponderados y evaluar su asociación con variables antropométricas y raciales en niños y adolescentes sanos.

\section{Material y Método}

El presente estudio corresponde a una revisión sistemática de la literatura.

\section{Criterios de elegibilidad de los artículos primarios}

Se consideró estudios de corte transversal, realizados en niños y adolescentes de ambos géneros entre 6 y 18 años, publicados en inglés, español, francés y portugués. Los estudios debían reportar los valores de normalidad de la DR durante el TM6', características antropométricas y el país de procedencia de la muestra.

El TM6' debía ser desarrollado de acuerdo a las normas publicadas por la ATS; las que establecen las 
directrices respecto a indicaciones, contraindicaciones y protocolo de ejecución, incluyendo la distancia establecida en el circuito (30 metros) y la metodología para estimular al individuo durante las pruebas ${ }^{3}$.

Como criterio de exclusión fueron considerados aquellos artículos que agruparon datos de sujetos mayores de 18 años o menores de 6 años, sujetos con enfermedades crónicas u obesos sin análisis diferenciado de los individuos sanos y enfermos. Adicionalmente, fueron excluidos aquellos estudios en los cuales el TM6' se realizó considerando una distancia diferente de 30 metros y/o incorporando algún elemento adicional, no incluido en el consenso ATS, para el desarrollo del protocolo.

\section{Búsqueda de artículos}

La búsqueda de los artículos se realizó en 6 bases de datos: Medline (Pubmed); Scientific Electronic Library Online (SciELO); Academic Search Complete; Psychology and Behavioral Science Collection; Rehabilitation and Sports Medicine Source y CINAHL. Adicionalmente, fueron considerados los textos atingentes de las listas de referencia de los artículos primarios. Para la búsqueda se emplearon los términos descriptores médicos (Medical Subject Heading; Mesh, por sus siglas en inglés), términos libres y términos booleano; los cuales fueron combinados mediante la siguiente estrategia de búsqueda: (Healthy[Title/Abstract]) $O R$ children[Title/Abstract]) OR adolescents[Title/Abstract]) AND exercise test[Title/Abstract]) OR six minute walk test[Title/Abstract]) AND normal values[Title/ Abstract]) OR reference values[Title/Abstract]. Solo fue empleado un filtro específico para la edad (Child: birth-18 years). En la búsqueda fueron considerados estudios desde su ingreso a la base de datos hasta el mes de junio de 2017.

\section{Identificación de los artículos}

Tres investigadores de forma independiente (F.M., B.C. y C.F) revisaron los títulos y los resúmenes de los artículos atingentes. Luego se obtuvieron los textos extensos de los resúmenes que cumplieron con los criterios de elegibilidad.

Los datos fueron extraídos y registrado en una planilla ad-hoc de manera independiente por 3 autores de la revisión (F.M., B.C. y C.F). Se registró año y lenguaje de publicación, nacionalidad de los sujetos de estudio, tamaño de la muestra, edad y género de los participantes, distancia recorrida durante el TM6 $6^{\prime}$ y, cuando se efectuó análisis de regresión, se registró la magnitud de la correlación entre la DR con las variables predictivas.

\section{Calidad metodológica de los artículos primarios}

La calidad metodológica fue evaluada por dos investigadores de forma independiente (FM y BC), a través de la verificación del cumplimiento de los ítems de la iniciativa STROBE (Strengthening the Reporting of Observational Studies in Epidemiology) ${ }^{22}$. Este instrumento fue construido para establecer las secciones que deben ser consideradas en el reporte de un estudio con diseño observacional. Si bien es cierto la iniciativa STORBE no fue creada para determinar el riesgo de sesgo, ha sido empleada para evaluar este atributo en otras revisiones sistemáticas de estudios observacionales del área de la rehabilitación y medicina física ${ }^{23}$.

\section{Análisis estadístico}

Los promedios de los valores normales de la DR durante el TM6' y las desviaciones estándar fueron ponderadas de acuerdo al tamaño muestral con el fin de estimar la DR global ponderada. Por su parte, las variables cualitativas fueron expresadas en valores absolutos y porcentajes.

Mediante análisis de varianza (ANOVA) de un factor se evaluó la significancia de la diferencia en la DR ponderada entre grupos etarios, así como también, entre los continentes de procedencia. Este análisis fue realizado en el software estadístico GraphPad Prism version 5.00, (GraphPad Software, San Diego California USA).

Finalmente, los coeficientes de correlación (CC) entre la distancia caminada durante el test de caminata de 6 min y las co-variables: edad, peso y estatura; fueron metaanalizados de acuerdo al método de Hedges-Olkin ${ }^{24}$, en el cual, son calculados los intervalos de confianza de los CC a través de la transformación z de Fisher. Por su parte, la inconsistencia fue calculada a través del estadístico $\mathrm{I}^{2}$ como $\mathrm{I}^{2}=100 \%(\mathrm{Q}-\mathrm{df}) / \mathrm{Q}$ en el que $\mathrm{Q}$ es el índice de heterogeneidad de Cochran y df, grados le libertad. Un valor de $0 \%$ indica la inexistencia de heterogeneidad y valores mayores indican existencia de heterogeneidad. El metaanálisis fue realizado utilizando el modelo de efectos aleatorios en el software MedCalc Statistical Software version 16.8.4 (MedCalc Software bvba, Ostend, Belgium).

En todos los análisis fue considerado un valor de $\mathrm{p}<0,05$ como significativo.

\section{Resultado}

\section{Selección de los estudios}

La búsqueda arrojó 11.341 artículos desde las 6 bases de datos. En la figura 1, se describe la secuencia de búsqueda sistemática y las razones de la exclusión. Un total de 10 artículos se consideraron para la revisión (figura 1).

\section{Características de los estudios}

Nueve artículos fueron escritos en inglés $711-16,18,19$ y 1 artículo fue escrito en español ${ }^{17}$. El rango de años 


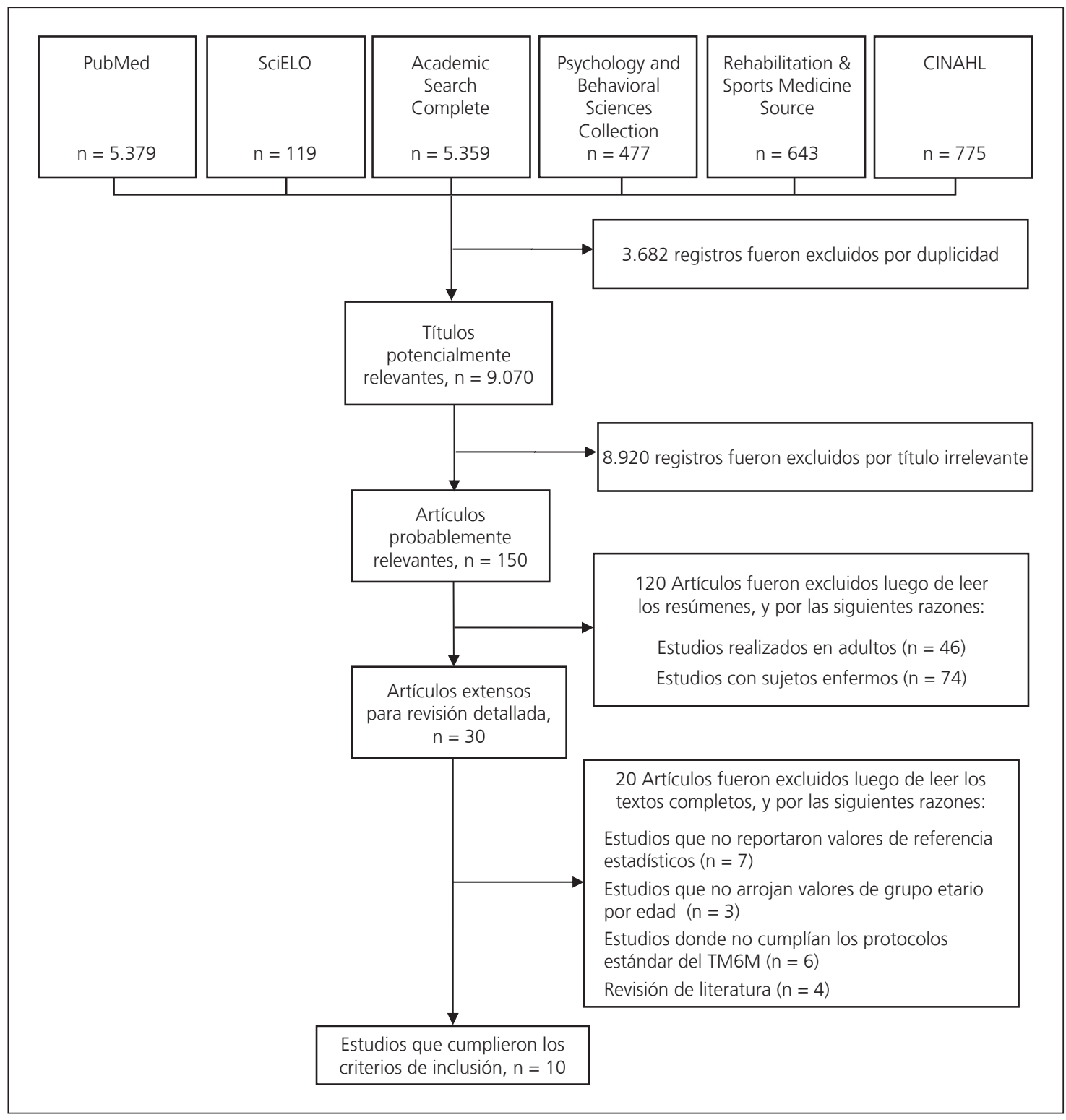

Figura 1. Secuencia de búsqueda sistemática de los artículos atingentes.

de publicación fue 2007 y 2015. El tamaño muestral encontrado en los estudios fue entre 100 y 1.445 ; un total de 5.352 completaron el TM6' en los 10 estudios; $2.783(51,9 \%)$ fueron hombres y $2.569(48,0 \%)$ fueron mujeres. En 7 artículos la muestra provino de Asia $(\mathrm{n}=4.514 ; 84,3 \%)^{7,12,14-16,18,19}$, en 2 de América del Sur $(\mathrm{n}=380 ; 7,1 \%)^{13,17} \mathrm{y}$ en 1 de Europa $(\mathrm{n}=458 ; 8,5 \%)^{11}$. En 3 estudios la edad mínima considerada fue 6 años $(\mathrm{n}=838 ; 15,6 \%)^{11,13,17}, 4$ estudios 7 años $(\mathrm{n}=2.726$; $50,9 \%)^{14,15,18,19}, 2$ estudios 9 años $(\mathrm{n}=839 ; 15,6 \%)^{7,12}$ y 1 estudio 12 años $(n=949 ; 17,7 \%)^{16}$. Por su parte, en 4 estudios la edad máxima estudiada fue 12 años $(\mathrm{n}=1427 ; 26,6 \%)^{7,12,13,18}, 1$ estudio 14 años $(\mathrm{n}=192$; $3,6 \%)^{17}$, 3 estudios 16 años $(\mathrm{n}=2022 ; 37,7 \%)^{11,14,15}, 1$ estudio 17 años $(\mathrm{n}=762 ; 14,2 \%)^{19}$ y 1 estudio 18 años $(\mathrm{n}=949 ; 17,7 \%)^{16}$.
En 6 estudios fue reportada una ecuación y/o una curva predictiva para la estimación del valor normal de la distancia caminada durante el TM6 ${ }^{11,13,15-17,19}$. En la tabla 1 se muestran las características generales de los artículos primarios.

\section{Riesgo de sesgo de los estudios}

Todos los artículos primarios cumplieron entre 14 y 20 ítems de la declaración STROBE. La mediana de cumplimiento fue de 17,0 (77,3\%) (tabla 2).

\section{Valores normales de la DR durante el TM6'}

En 6 estudios fue reportado el promedio general de la DR para ambos géneros separadamente $(\mathrm{n}=3.638$; $56,0 \%)$ y en 8 fue reportada la DR de forma agrupada $(\mathrm{n}=3.945 ; 73,7 \%)$. El promedio ponderado de la DR 
Tabla 1. Características de los artículos primarios

\begin{tabular}{|c|c|c|c|c|c|c|c|c|}
\hline \multirow[t]{2}{*}{ Autor } & \multirow[t]{2}{*}{ País } & \multicolumn{4}{|c|}{ Protocolo TM6' } & \multirow{2}{*}{$\begin{array}{l}\mathrm{N} \text { total } \\
(\mathrm{H} / \mathrm{M})\end{array}$} & \multirow{2}{*}{$\begin{array}{c}\text { Edad } \\
\text { (Rango) }\end{array}$} & \multirow{2}{*}{$\begin{array}{l}\text { Reporte de } \\
\text { ecuaciones } \\
\text { y/o curvas } \\
\text { predictivas }\end{array}$} \\
\hline & & $\begin{array}{c}\text { Período de } \\
\text { inactividad } \\
\text { antes del TM6 }\end{array}$ & Distancia & $\begin{array}{c}\text { Variables } \\
\text { consideradas }\end{array}$ & $\begin{array}{c}\text { Instrucción/ } \\
\text { Estímulo }\end{array}$ & & & \\
\hline $\begin{array}{l}\text { Li, A., et al. } \\
(2007)\end{array}$ & China & $10 \mathrm{~min}$ & $30 \mathrm{~m}$ & $\mathrm{DR}$, Sat $\mathrm{O}_{2}, \mathrm{FC}$ & $\begin{array}{l}\text { Estándar } \\
\text { ATS }\end{array}$ & $\begin{array}{c}1.445 \\
(805-640)\end{array}$ & $7-16$ & $\begin{array}{c}\text { Ecuaciones y } \\
\text { curvas }\end{array}$ \\
\hline $\begin{array}{l}\text { Priesnitz, C., et al. } \\
\text { (2009) }\end{array}$ & Brasil & ND & $30 \mathrm{~m}$ & $\begin{array}{l}\text { DR, Sat O2, } \\
F C, P A, P E\end{array}$ & $\begin{array}{l}\text { Estándar } \\
\text { ATS }\end{array}$ & $\begin{array}{c}188 \\
(92-96)\end{array}$ & $6-12$ & Ecuaciones \\
\hline $\begin{array}{l}\text { Limsuwan, A., et al. } \\
(2010)\end{array}$ & Tailandia & $30-60 \mathrm{~min}$ & $30 \mathrm{~m}$ & $D C, F C$ & $\begin{array}{l}\text { Estándar } \\
\text { ATS }\end{array}$ & $\begin{array}{c}100 \\
(53-47\end{array}$ & $9-12$ & No \\
\hline $\begin{array}{l}\text { Tonklang, N., et al. } \\
\text { (2011) }\end{array}$ & Tailandia & $10 \mathrm{~min}$ & $30 \mathrm{~m}$ & $\begin{array}{l}\text { DR, FC, FR, } \\
\text { PE, PA }\end{array}$ & $\begin{array}{l}\text { Estándar } \\
\text { ATS }\end{array}$ & $\begin{array}{c}739 \\
(403-336)\end{array}$ & $9-12$ & No \\
\hline $\begin{array}{l}\text { D'Silva, C., et al. } \\
\text { (2012) }\end{array}$ & India & $10 \mathrm{~min}$ & $30 \mathrm{~m}$ & $\begin{array}{l}\text { DR, Sat O2, } \\
\text { FC, PA, PE }\end{array}$ & $\begin{array}{l}\text { Estándar } \\
\text { ATS }\end{array}$ & $\begin{array}{c}400 \\
(202-198)\end{array}$ & $7-12$ & No \\
\hline $\begin{array}{l}\text { Gatica, D., et al. } \\
(2012)\end{array}$ & Chile & $10 \mathrm{~min}$ & $30 \mathrm{~m}$ & $\begin{array}{l}\text { DR, Sat O2, } \\
\quad \text { FC, PE }\end{array}$ & $\begin{array}{l}\text { Estándar } \\
\text { ATS }\end{array}$ & $\begin{array}{c}192 \\
(92-100)\end{array}$ & $6-14$ & Ecuaciones \\
\hline $\begin{array}{l}\text { Ulrich, S., et al. } \\
\text { (2013) }\end{array}$ & Suiza & $5 \mathrm{~min}$ & $30 \mathrm{~m}$ & $\begin{array}{l}\text { DR, Sat O2, } \\
\text { FC, PA }\end{array}$ & $\begin{array}{l}\text { Estándar } \\
\text { ATS }\end{array}$ & $\begin{array}{c}458 \\
(225-233)\end{array}$ & $6-16$ & $\begin{array}{c}\text { Ecuaciones y } \\
\text { curvas }\end{array}$ \\
\hline $\begin{array}{l}\text { Kanburoglu, M., et al. } \\
\text { (2014) }\end{array}$ & Turquía & $5 \mathrm{~min}$ & $30 \mathrm{~m}$ & $\begin{array}{l}\mathrm{DR}, \mathrm{FC}, \mathrm{PA} \\
\mathrm{PE}\end{array}$ & $\begin{array}{l}\text { Estándar } \\
\text { ATS }\end{array}$ & $\begin{array}{c}949 \\
(467-482)\end{array}$ & $12-18$ & Curvas \\
\hline $\begin{array}{l}\text { Prajapati, N., et al } \\
\text { (2014) }\end{array}$ & India & ND & $30 \mathrm{~m}$ & $\mathrm{DR}, \mathrm{FC}$ & $\begin{array}{l}\text { Estándar } \\
\text { ATS }\end{array}$ & $\begin{array}{c}119 \\
(62-57)\end{array}$ & $7-16$ & No \\
\hline $\begin{array}{l}\text { Chen, Ch., et al. } \\
\text { (2015) }\end{array}$ & Taiwán & ND & $30 \mathrm{~m}$ & $\begin{array}{c}\mathrm{DR}, \mathrm{FC} \text {, Sat } \\
\mathrm{O}_{2}\end{array}$ & $\begin{array}{l}\text { Estándar } \\
\text { ATS }\end{array}$ & $\begin{array}{c}762 \\
(382-380)\end{array}$ & $7-17$ & Curvas \\
\hline
\end{tabular}

ND: No declarado; DR: Distancia recorrida durante el TM6'; Sat $\mathrm{O}_{2}$ : FC: Frecuencia cardiaca; PA: Presión arterial; PE: Percepción de esfuerzo; FR: Frecuencia respiratoria; Hombre; M: Mujer.

\section{Tabla 2. Lista de verificación STROBE}

\begin{tabular}{|c|c|c|c|c|c|c|c|c|c|c|c|c|c|c|c|c|c|c|c|c|c|c|c|}
\hline Autor & 1 & 2 & 3 & 4 & 5 & 6 & 7 & 8 & 9 & 10 & 11 & 12 & 13 & 14 & 15 & 16 & 17 & 18 & 19 & 20 & 21 & 22 & Total \\
\hline $\begin{array}{l}\text { Li, A., et al. } \\
(2007)\end{array}$ & 1 & 1 & 1 & 0 & 1 & 1 & 1 & 1 & 0 & 1 & 1 & 1 & 1 & 1 & 1 & 1 & 1 & 0 & 0 & 1 & 0 & 1 & $17(77,3 \%)$ \\
\hline $\begin{array}{l}\text { Priesnitz, C., et } \\
\text { al. (2009) }\end{array}$ & 1 & 1 & 1 & 1 & 1 & 1 & 1 & 1 & 0 & 1 & 1 & 1 & 1 & 1 & 1 & 1 & 1 & 1 & 0 & 1 & 0 & 0 & $18(81,8 \%)$ \\
\hline $\begin{array}{l}\text { Limsuwan, A., } \\
\text { et al. (2010) }\end{array}$ & 1 & 0 & 1 & 0 & 1 & 0 & 1 & 1 & 0 & 0 & 1 & 1 & 1 & 1 & 1 & 0 & 0 & 1 & 1 & 1 & 1 & 0 & $14(63,6 \%)$ \\
\hline $\begin{array}{l}\text { Tonklang, N., } \\
\text { et al. (2011) }\end{array}$ & 1 & 1 & 1 & 0 & 1 & 1 & 1 & 1 & 0 & 0 & 0 & 1 & 1 & 1 & 1 & 1 & 1 & 1 & 0 & 1 & 0 & 0 & $15(68,2 \%)$ \\
\hline $\begin{array}{l}\text { D'Silva, C., et } \\
\text { al. (2012) }\end{array}$ & 1 & 1 & 1 & 0 & 1 & 1 & 1 & 1 & 0 & 0 & 0 & 0 & 1 & 1 & 1 & 1 & 1 & 0 & 0 & 1 & 0 & 1 & $14(63,6 \%)$ \\
\hline $\begin{array}{l}\text { Gatica, D., et } \\
\text { al. (2012) }\end{array}$ & 1 & 1 & 1 & 1 & 1 & 1 & 1 & 1 & 0 & 0 & 1 & 1 & 1 & 1 & 1 & 1 & 1 & 1 & 0 & 1 & 0 & 0 & $17(77,3 \%)$ \\
\hline $\begin{array}{l}\text { Ulrich, S., et } \\
\text { al. (2013) }\end{array}$ & 1 & 1 & 1 & 0 & 1 & 1 & 0 & 1 & 0 & 1 & 1 & 1 & 1 & 1 & 1 & 1 & 1 & 1 & 1 & 1 & 1 & 1 & $19(86,4 \%)$ \\
\hline $\begin{array}{l}\text { Kanburoglu, } \\
\text { M., et al. } \\
(2014)\end{array}$ & 1 & 1 & 1 & 1 & 1 & 0 & 1 & 1 & 0 & 1 & 1 & 1 & 1 & 1 & 1 & 1 & 1 & 0 & 1 & 1 & 1 & 1 & $19(86,4 \%)$ \\
\hline $\begin{array}{l}\text { Prajapati, N., } \\
\text { et al (2014) }\end{array}$ & 1 & 1 & 1 & 1 & 1 & 1 & 1 & 1 & 0 & 0 & 0 & 0 & 1 & 1 & 1 & 1 & 0 & 0 & 1 & 1 & 0 & 0 & $14(63,6 \%)$ \\
\hline $\begin{array}{l}\text { Chen, Ch., et } \\
\text { al. (2015) }\end{array}$ & 1 & 1 & 1 & 0 & 1 & 1 & 1 & 1 & 0 & 1 & 1 & 1 & 1 & 1 & 1 & 1 & 1 & 1 & 1 & 1 & 1 & 1 & $20(90,9 \%)$ \\
\hline
\end{tabular}




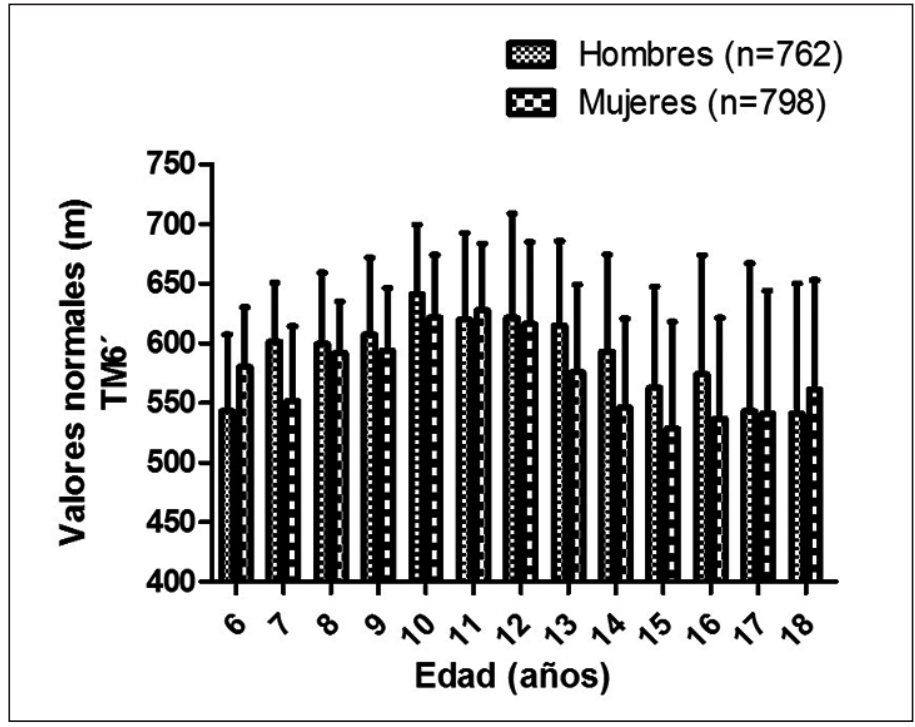

Figura 2. Valores normales del test de marcha de 6 min en función de la edad. TM6': Test de marcha de 6 minutos. Resultados se expresan en promedio ponderado según tamaño muestral.

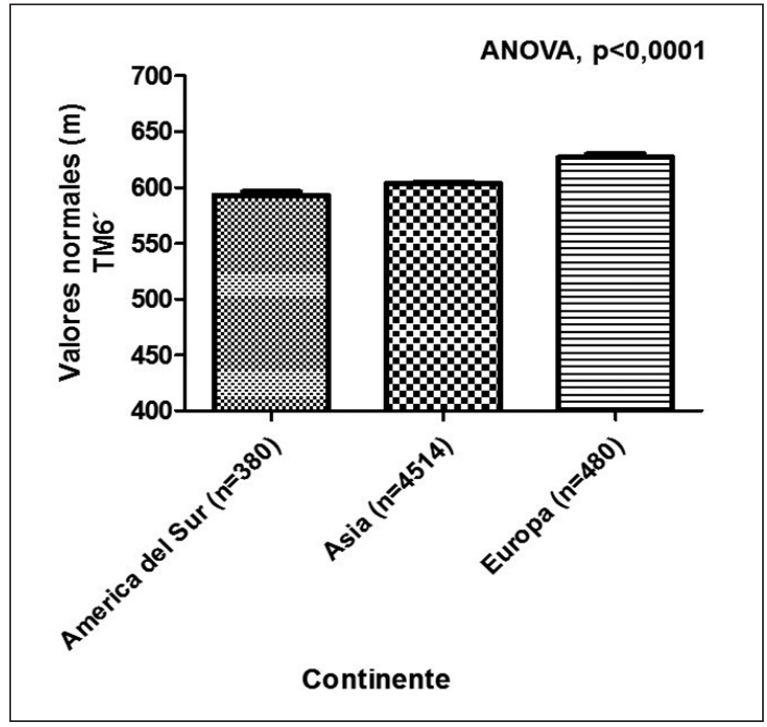

Figura 3. Valores normales del test de marcha de 6 min en función del continente de procedencia de los valores predictivos. TM6': Test de marcha de 6 minutos. en los hombres fue de $672,8 \pm 66,1 \mathrm{~m}$ y en las mujeres fue de $623,4 \pm 53,2 \mathrm{~m}(\mathrm{p}<0,0001)^{7,12,14,15,17,18}$. Por su parte, el promedio ponderado de la DR considerando los valores de ambos géneros agrupados fue de 619,8 \pm $58,3 \mathrm{~m}^{7,12-15,17-19}$.

En la figura 2, se grafican los valores normales de la DR según edad. En los hombres el análisis de varianza determinó que los niños de 6 años caminaron significativamente menos distancia que aquellos de edades entre 10 y 13 años. Los niños de 10, 11 y 12 años caminaron una mayor distancia comparado a aquellos entre 15 a 18 años $(\mathrm{p}<0,0001)$. Por su parte, en las mujeres, el análisis de varianza determinó que las niñas de 7 años caminaron una menor distancia que aquellas de 10 a 12 años y las niñas de 10, 11 y 12 años caminaron una mayor distancia comparado a aquellas entre 14 y 17 años. Adicionalmente, existió diferencia significativa de la DR entre las niñas de 8, 9, 15 y 16 años $(\mathrm{p}<0,0001)$.

Por otra parte, se observó diferencia significativa de la DR ponderada entre los continentes de procedencia de los estudios analizados. Así, mientras que en Sudamérica la DR ponderada fue de 593,1 $\pm 64,3$; en Asia y Europa fue de $603,7 \pm 78,9$ y $627,2 \pm 61,1$ respectivamente $(\mathrm{p}<0,0001)$ (figura 3$)$.

\section{Correlación entre la DR durante el TM6' y variables asociadas}

La figura 4 (A, B y C) muestra los gráficos de bosque de los coeficientes agrupados de la correlación entre la DR con la edad, estatura y peso. El metaanálisis de efectos aleatorios reveló la existencia de correlación positiva significativa entre el rendimiento durante el TM6 y la edad $(r=44 ; 0,28-0,58)$, estatura $(0,47 ; 0,33$ $0,60)$ y peso $(0,40 ; 0,17-0,60)$ de los sujetos estudiados (tabla 3).

\section{Discusión}

El presente estudio pretendió analizar los valores de referencia de la DR durante el TM6' ejecutado bajo el estándar ATS, con el fin de establecer un rango de normalidad global, así como también, determinar las variables asociadas al rendimiento durante esta prueba en niños y adolescentes sanos. A diferencia de revisiones sistemáticas previamente publicadas ${ }^{2,6}$, en nuestro estudio solo se consideró estudios donde el TM6' se realizó bajo las normas ATS; no obstante, la magnitud de la heterogeneidad entre los estudios no permitió establecer valores predictivos agrupados para cada grupo etario. En este contexto, pese a que el análisis de varianza reveló que la DR muestra diferencias significativas entre los grupos de edad, estas diferencias no fueron apreciadas entre edades consecutivas. Vale la pena destacar que la DR mostró un incremento gradual hasta los 10 y 12 años en hombres y las mujeres respectivamente, para luego revelar un comportamiento independiente de la edad hasta los 18 años. Estas observaciones concuerdan con lo reportado en el estudio de Ulrich y cols, en el cual observaron patrones de asociación diferenciados entre preadolescentes y 
Figura 4. Gráficos de bosque de los estudios que reportan el metaanálisis de la correlación entre la distancia recorrida durante el TM6' y las variables predictoras. En A correlación con la edad, en B correlación con la estatura y en $\mathbf{C}$ correlación con el peso de los sujetos estudiados.



Tabla 3. Metaanálisis de efectos aleatorios de la correlación entre la distancia caminada durante el TM6' y variables predictoras

\begin{tabular}{cccccc}
\hline Co-variable & $N$ & $K$ & r ponderado $(95 \%$ IC) & Valor $p$ & $1^{2}$ \\
\hline Edad & 3.293 & 6 & $0,44(0,28-0,58)$ & $<0,001$ & 95,9 \\
Estatura & 3.393 & 7 & $0,47(0,33-0,60)$ & $<0,001$ & 95,3 \\
Peso & 2.344 & 5 & $0,40(0,17-0,60)$ & 0,001 & 96,4 \\
\hline
\end{tabular}

n: Número de sujetos; k: Número de artículos; 12: \% de inconsistencia.

adolescentes, lo cual, fue refrendado con la generación de ecuaciones predictivas independientes para ambos grupos de edad ${ }^{11}$.

Por otra parte, el metaanálisis de efectos aleatorios reveló tan solo una moderada correlación entre las co-variables edad, peso y estatura con la DR durante el TM6'. Indicando que estas variables (ampliamente consideradas como variables predictoras en las ecuaciones de regresión) determinan escasamente la DR durante esta prueba (entre $16,0 \%$ y $22,1 \%$ ). Este comportamiento ha sido observado en otras pruebas funcionales como la fuerza muscular inspiratoria, en la cual, se ha cuestionado la asociación entre la magnitud de las variaciones inducidas por el crecimiento en los parámetros antropométricos y los cambios en la presión inspiratoria máxima en niños y adolescentes ${ }^{25}$.

En relación al análisis por género, fue posible establecer una diferencia ponderada de 51,0 m, siendo superior en hombres respecto a las mujeres. Esto concuerda con los resultados observados en otras pruebas de campo ${ }^{26,27}$ y es respaldado fuertemente por las diferencias de composición corporal existentes entre géne$\operatorname{ros}^{28}$, lo que condiciona la brecha de capacidad física entre hombres y mujeres ${ }^{29}$.

De manera interesante fue posible establecer diferencias significativas entre los continentes de procedencia de los artículos primarios. Esta observación confirma lo que han sugerido otros estudios, en los cuales, se menciona el posible impacto de las características raciales de los habitantes de los distintos continentes sobre el rendimiento durante el $\mathrm{TM}^{2}{ }^{2}$. La variabilidad en la DR asociada al país de procedencia probablemente se asocia a las diferencias en las características antropométricas entre los habitantes de los distintos continentes ${ }^{30}$. En este sentido, algunos estudios sugieren que la longitud de las extremidades inferiores y la estatura son las variables que mejor determinan la DR normal en niños ${ }^{31}$, lo que concuerda con lo observado en el metaanálisis de efectos aleatorios realizado en nuestro estudio (tabla 3 ). Por lo tanto, es probable 
que la diferencia en la estatura entre los habitantes de los distintos continentes haya afectado los valores normales de la DR durante el TM6'.

Vale la pena destacar que en las revisiones sistemáticas previamente realizadas se desarrolló una metodología de análisis y reporte de los resultados basado en un enfoque cualitativo, lo cual, solo le confirió un alcance descriptivo a sus conclusiones ${ }^{2,6}$. Por el contrario, en nuestro estudio fue posible analizar los valores normales de la DR durante el TM6' y su asociación con otras variables predictores mediante un abordaje metaanalítico, permitiendo la verificación de algunas hipótesis con una confianza de magnitud conocida.

Nuestro estudio posee ciertas debilidades que deben ser discutidas. El metaanálisis de efectos aleatorios arrojó un elevado nivel de heterogeneidad, lo cual, no permite descartar que la magnitud de asociación estimada haya sido influenciada por la existencia de error aleatorio y los sesgos observados en los estudios. Vale la pena destacar que, para evitar acrecentar la variabilidad entre los artículos primarios, en el presente trabajo no fueron considerados estudios en que el protocolo del TM6' no fuera ajustado a las recomendaciones establecidas por la ATS, sugiriendo que las diferencias entre las características poblacionales, así como posibles diferencias metodológicas en el registro de datos, pudieron haber sido los principales factores asociados con la alta heterogeneidad observada en el metaanálisis.

Finalmente es posible concluir que la DR durante el TM6' muestra asociación significativa con el género y continente de procedencia del estudio. Por su parte, la estatura, el peso y la edad muestran una moderada asociación con la DR determinando un bajo porcentaje de la DR normal ideal en niños y adolescentes sanos, lo cual, debe ser considerado para la construcción de valores de referencia. Por su parte, futuros estudios lon- gitudinales deben ser ejecutados para confirmar estos resultados.

\section{Responsabilidades éticas}

Protección de personas y animales: Los autores declaran que los procedimientos seguidos se conformaron a las normas éticas del comité de experimentación humana responsable y de acuerdo con la Asociación Médica Mundial y la Declaración de Helsinki.

Confidencialidad de los datos: Los autores declaran que han seguido los protocolos de su centro de trabajo sobre la publicación de datos de pacientes.

Derecho a la privacidad y consentimiento informado: Los autores han obtenido el consentimiento informado de los pacientes y/o sujetos referidos en el artículo. Este documento obra en poder del autor de correspondencia.

\section{Financiación}

Este estudio fue financiado por la escuela de Kinesiología de la Universidad San Sebastián, Concepción, Chile.

\section{Conflicto de intereses}

Los autores declaran no tener conflicto de intereses.

\section{Agradecimientos}

En memoria del kinesiólogo Darwin Gatica Solís (Q.E.P.D) por su valiosa contribución al desarrollo de este artículo y a la rehabilitación cardiopulmonar infantil de Chile.

\section{Referencias}

1. Bartels B, de Groot JF, Terwee CB. The six-minute walk test in chronic pediatric conditions: a systematic review of measurement properties. Phys Ther. 2013;93(4):529-41.

2. Mylius CF, Paap D, Takken T. Reference value for the 6-minute walk test in children and adolescents: a systematic review. Expert Rev Respir Med. 2016;10(12):1335-52.

3. ATS statement: guidelines for the sixminute walk test. Am J Respir Crit Care Med. 2002;166(1):111-7.

4. Rodríguez I, Heríquez S, Vásquez $\mathrm{P}$, Zenteno D. Test de caminata de seis minutos y función pulmonar en pacientes con bronquiolitis obliterante post infecciosa. Rev Chil Enferm Respir. 2014;30(2):68-74.

5. Andrade LB, Silva DA, Salgado TL, Figueroa JN, Lucena-Silva N, Britto MC. Comparison of six-minute walk test in children with moderate/severe asthma with reference values for healthy children. J Pediatr (Rio J). 2014;90(3):250-7.

6. Cacau LA, Santana-Filho VJ, Maynard LG, Gomes MN, Fernandes M, Carvalho VO. Reference Values for the Six-Minute Walk Test in Healthy Children and Adolescents: a Systematic Review. Braz J Cardiovasc Surg. 2016;31(5):381-8.

7. Limsuwan A, Wongwandee R, Khowsathit P. Correlation between 6-min walk test and exercise stress test in healthy children.
Acta Paediatr. 2010;99(3):438-41.

8. Zuk M, Migdal A. Six-Minute Walk Test in Evaluation of Children with Pulmonary Arterial Hypertension. 2017.

9. Jagiellowicz-Kowalska D, Mazurkiewicz K, Sadel-Wieczorek A, Brzezinska-Rajszys G, Donadio MV, Heinzmann-Filho JP, et al. Six-Minute Walk Test Results Predict Risk of Hospitalization for Youths with Cystic Fibrosis: A 5-Year Follow-Up Study. Pediatr Cardiol. 2017;182:204-9.e1.

10. den Boer SL, Flipse DH, van der Meulen MH, Backx AP, du Marchie Sarvaas GJ, Ten Harkel AD, et al. Six-Minute Walk Test as a Predictor for Outcome in Children with Dilated Cardiomyopathy and Chronic Stable Heart Failure. Pediatr Cardiol. 2017;38(3):465-71. 
11. Ulrich S, Hildenbrand FF, Treder U, Fischler M, Keusch S, Speich R, et al. Reference values for the 6-minute walk test in healthy children and adolescents in Switzerland. BMC Pulm Med. 2013;13:49.

12. Tonklang N, Roymanee S, Sopontammarak S. Developing standard reference data for Thai children from a six-minute walk test. J Med Assoc Thai. 2011;94(4):470-5.

13. Priesnitz CV, Rodrigues GH, Stumpf Cda S, Viapiana G, Cabral CP, Stein RT, et al. Reference values for the 6-min walk test in healthy children aged 6-12 years. Pediatr Pulmonol. 2009;44(12):1174-9.

14. Namrata P, Anjali B. A Study to Know the Average Value of Six Minute Walk Distance Test in Children Aged 7 to 16 Years of Ahmedabad City. Indian Journal of Physiotherapy and Occupational Therapy-An International Journal. 2014;8(1):49-53.

15. Li AM, Yin J, Au JT, So HK, Tsang T, Wong E, et al. Standard reference for the six-minute-walk test in healthy children aged 7 to 16 years. Am J Respir Crit Care Med. 2007;176(2):174-80.

16. Kanburoglu MK, Ozdemir FM, Ozkan S, Tunaoglu FS. Reference values of the 6-minute walk test in healthy Turkish children and adolescents between 11 and 18 years of age. Respir Care. 2014;59(9):1369-75.

17. Gatica D, Puppo H, Villarroel G, San Martín I, Lagos R, Montecino JJ, et al. Reference values for the 6-minutes walking test in healthy Chilean children. Rev Med Chile 2012;140(8):1014-21.

18. D'Silva C, Vaishali K, Venkatesan P.
Six-minute walk test-normal values of school children aged 7-12 y in India: a cross-sectional study. Indian J Pediatr. 2012;79(5):597-601.

19. Chen CA, Chang CH, Lin MT, Hua YC, Fang WQ, Wu MH, et al. Six-Minute Walking Test: Normal Reference Values for Taiwanese Children and Adolescents. Acta Cardiol Sin. 2015;31(3):193-201.

20. Goemans N, Klingels K, van den Hauwe M, Boons S, Verstraete L, Peeters $\mathrm{C}$, et al. Six-minute walk test: reference values and prediction equation in healthy boys aged 5 to 12 years. PLoS One. 2013;8(12):e84120.

21. Geiger R, Strasak A, Treml B, Gasser K, Kleinsasser A, Fischer V, et al. Six-minute walk test in children and adolescents. J Pediatr. 2007;150(4):395-9, 9.e1-2.

22. von Elm E, Altman DG, Egger M, Pocock SJ, Gotzsche PC, Vandenbroucke JP. The Strengthening the Reporting of Observational Studies in Epidemiology (STROBE) statement: guidelines for reporting observational studies. Ann Intern Med. 2007;147(8):573-7.

23. Stanton TR, Leake HB, Chalmers KJ, Moseley GL. Evidence of Impaired Proprioception in Chronic, Idiopathic Neck Pain: Systematic Review and Meta-Analysis. Phys Ther. 2016;96(6):876-87.

24. Rodríguez I, Zambrano L, Manterola C. Criterion-related validity of perceived exertion scales in healty children: a systematic review and meta-analysis. Arch Argent Pediatr. 2016;114(1).

25. Harik-Khan RI, Wise RA, Fozard JL. Determinants of maximal inspiratory pressure. The Baltimore Longitudinal Study of Aging. Am J Respir Crit Care Med. 1998;158(5 Pt 1):1459-64.

26. Rodríguez-Núñez I, Gatica Solís D, Navarro Tapia X. Rendimiento de las variables cardiorrespiratorias durante el test de Chester en niños sanos chilenos. Revista Cubana de Pediatría. 2016;88:31022.

27. Lanza Fde C, Zagatto Edo P, Silva JC, Selman JP, Imperatori TB, Zanatta DJ, et al. Reference Equation for the Incremental Shuttle Walk Test in Children and Adolescents. J Pediatr. 2015;167(5):105761.

28. Bray GA, DeLany JP, Harsha DW, Volaufova J, Champagne CM. Body composition of African American and white children: a 2-year follow-up of the BAROC study. Obes Res. 2001;9(10):60521.

29. Lai N, Martis A, Belfiori A, TolentinoSilva F, Nasca MM, Strainic J, et al. Gender differences in $\mathrm{V} \mathrm{O} 2$ and $\mathrm{HR}$ kinetics at the onset of moderate and heavy exercise intensity in adolescents. Physiol Rep. 2016;4(18).

30. Rønn PF, Andersen GS, Lauritzen T, Christensen DL, Aadahl M, Carstensen B, et al. Ethnic differences in anthropometric measures and abdominal fat distribution: a cross-sectional pooled study in Inuit, Africans and Europeans. J Epidemiol Community Health. 2017;71(6):536-43.

31. Oliveira AC, Rodrigues CC, Rolim DS, Souza AA, Nascimento OA, Jardim JR, et al. Six-minute walk test in healthy children: is the leg length important? Pediatr Pulmonol. 2013;48(9):921-6. 